\title{
JOURNAL OF PHILOSOPHICAL STUDIES
}

the lines he has set forth . . . and to fall somewhere within the framework which he supplies." In conclusion, Professor Laird offers some reflections upon the cardinal points in the analysis ; namely, on the "ownership" of the objects of experience, the nature of the empirical self, and the unknowability of the pure ego or subject.

S. $\mathbf{K}$.

\section{CORRESPONDENCE}

READERS are informed that when a sufficient number of requests for bibliographical information upon a particular philosophical topic have been received, an endeavour will be made to deal with them collectively in the Journal by means of a short critical note. 